\title{
XVIII.
}

Aus dem pharmakologischen Institut der Universität Bonn.

\section{Die Reduction der Arsensäure durch Organsäfte.}

\author{
Von \\ C. Binz.
}

Das Ergebniss der vorhergehenden $\left.{ }^{1}\right)$ Untersuchungsreihe war:

„Arsenige Säure in schwach alkalischer Lösung wird unter dem Einflusse des frischen Saftes des Dünndarmes, der Milz und besonders der Leber in beträchtlicher Menge zu Arsensäure oxydirt."

Es wurde damit von Neuem bestätigt, was ich und H. Schulz früher bereits gezeigt hatten. Auch das, was wir hinsichtlich des umgekehrten Vorganges, der Reduction der Arsensäure, gefunden hatten, wollte ich einer ernenten Prüfung und Erweiterung unterziehen. Ich unternahm das gemeinschaftlich mit Dr. C. Laar und gebe hier unsere bisherigen Resultate.

\section{Versuch.}

$0 \mathrm{chsenblut} 150 \mathrm{ccm}$ defibrinirt, mit $15 \mathrm{ccm} 2 / 10$-Normalnatriumarseniatlösung $=0,345 \quad \mathrm{As}_{2} \mathrm{O}_{5}$ versetzt und 24 Stunden bei Körperwärme digerirt. Dialyse unter Zusatz von etwas Essigsäure 48 Stunden. Nach deren Ablauf war kein Fäulnissgeruch wahrzunehmen.

Direct ausgefüllt, stark sauer

und in Eiskühlung, $23 \mathrm{mg} \mathrm{As} 2 \mathrm{~S}_{3}$

$$
=18,5 \quad \mathrm{As}_{2} \mathrm{O}_{3}=21,5 \mathrm{As}_{2} \mathrm{O}_{5}
$$

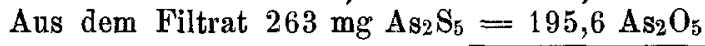

zusammen $217,1 \quad \mathrm{As}_{2} \mathrm{O}_{5}=62,9$ Proc. dialysirt.

Es wurden mithin redncirt 6,2 Proc. der angewandten Menge und 9,9 Proc. des dialysirten Antheiles.

\section{Versuch.}

Kalbsblut $150 \mathrm{cem}$ defibrinirt und ebenso wie im vorigen Versuche behandelt, nur unter starkem Zusatze von Chloroform dialysirt, um die Fäulniss zu verhïten.

1) Archiv f. exp. Path. u. Pharm. Bd. XXXVI. S. 275. 
Direct ausgefällt $7,2 \mathrm{mg} \mathrm{As} 2 \mathrm{~S}_{3}=5,8 \quad \mathrm{As}_{2} \mathrm{O}_{3}=6,7 \quad \mathrm{As}_{2} \mathrm{O}_{5}$. Das entspricht 1,9 Proc. reducirter Arsensäure von der angewandten Menge.

3. Versuch.

Kaninchenleber $104 \mathrm{~g}$, ganz frisch in der Maschine zerkleinert, angerïhrt mit $50 \mathrm{ccm}$ Wasser, digerirt $5 \frac{1}{2}$ Stunden bei Blatwärme mit $10 \mathrm{ccm}$ der Natriumarseniatlösung $=0,230 \mathrm{As}_{2} \mathrm{O}_{5}$. Dialyse ${ }^{1}$ ) unter $\mathrm{Zu}$ satz von Essigsäure bis zum stark sauren Geruch in einem küblen Raum während 40 Stunden.

Direct ausgefällt $110,2 \mathrm{mg} \mathrm{As} \mathrm{S}_{3}$

$$
=88,7 \mathrm{As}_{2} \mathrm{O}_{3}=103,0 \mathrm{As}_{2} \mathrm{O}_{5}
$$

Aus dem Filtrat $40,2 \mathrm{As}_{2} \mathrm{~S}_{5}=29,8 \mathrm{As}_{2} \mathrm{O}_{5}$

zusammen $132,8 \mathrm{As}_{2} \mathrm{O}_{5}=57,7$ Proc. dialysirt.

Es wurden reducirt 44,8 Proc. der angewandten Menge Arsensäure und 77,6 Proc. des dialysirten Antheiles.

\section{Versueh.}

Kaninchenleber $80 \mathrm{~g}$, wie vorher zerkleinert und unter Zusatz von $1 \mathrm{~g}$ in Weingeist gelöstem Thymol zum Abbalten der Fäulniss mit $50 \mathrm{cem}$ Wasser und $10 \mathrm{cem}$ Natriumarseniatlösung $=0,230 \mathrm{As}_{2} \mathrm{O}_{5}$ durch 6 Stunden digerirt. Dialyse in Britschrank 21 Stunden.

Direct ausgefällt $27,4 \mathrm{mg} \mathrm{As} 2 \mathrm{~S}_{3}$

$$
=22,1 \mathrm{As}_{2} \mathrm{O}_{3}=25,6 \mathrm{As}_{2} \mathrm{O}_{5}
$$

Aus dem Filtrat 194,3 $\mathrm{As}_{2} \mathrm{~S}_{5}=144,2 \mathrm{As}_{2} \mathrm{O}_{5}$

zusammen $169,8 \mathrm{As}_{2} \mathrm{O}_{5}=73,8$ Proc. dialysirt.

Es wurden reducirt 11,1 Proc. der angewandten Menge und 15,1 Proc. des dialysirten Antheiles.

\section{Versuch.}

Kaninchenleber $130 \mathrm{~g}$, wie bisher. Zusatz von $60 \mathrm{ccm}$ Wasser und von $1,25 \mathrm{~g}$ zerriebenem Thymol. Sodann $10 \mathrm{~cm}$ Natriumarseniatlösung. Der Brei mit Kohlensäure gesättigt und 6 Stunden digerirt. Dialyse bei Körperwärme durch 24 Stunden.

In den vorigen Versuchen hatte der Brei eine sanere Reaction angenommen; diesmal wurde er, um die Reduction bei den verschiedensten Zuständen zu untersuchen, durch Beigabe von Natriumbicarbonat schwach alkalisch gehalten, gleichzeitig einige Male Kohlensäure in die Dialysatorglocke eingeleitet und der Brei damit gemischt.

Direct ausgefällt $86,1 \mathrm{mg} \mathrm{As} 2 \mathrm{~S}_{3}$

$$
=69,3 \mathrm{As}_{2} \mathrm{O}_{3}=80,5 \mathrm{As}_{2} \mathrm{O}_{5}
$$

Aus dem Filtrat $87,4 \mathrm{As}_{2} \mathrm{~S}_{3}=64,8 \mathrm{As}_{2} \mathrm{O}_{5}$

$$
\text { zusammen } 145,3 \quad \mathrm{As}_{2} \mathrm{O}_{5}=63,2 \text { Proc. dialysirt. }
$$

Es wurden reducirt 35,0 Proc. der angewandten Menge und 55,4 Proc. des dialysirten Antheiles.

1) Als Dialysatoren wurden zuletzt immer die von Beckmann, Zeitschrift f. analyt. Chemie 1896. Bd. XXXV. S. 269, angegebenen sehr bequemen Pergamentpapier-Faltenfilter benutzt. 


\section{Versuch.}

Kaninchenleber, wie immer dem soeben durch Verbluten getödteten Thier entnommen, wurde nur grob mit der Scheere zerschnitten, in eine körperwarme Mischung von $5 \mathrm{ccm}$ der Natriumarseniatlösung und $50 \mathrm{ccm}$ Wasser eingetragen und damit ein e Stunde digerirt. Dann wurde rasch abfiltrirt, das Filtrat mit starker Salzsäure versetzt, der dadurch entstandene Niederschlag abfiltrirt, das ziemlich klare Filtrat mit Natriumbicarbonat eben ubbersättigt und dann 24 Stunden lang gegen die dreifache Menge Aussenwasser dialysirt. Das mit Salzsäure vermischte Dialysat gab mit Schwefelwasserstoff in Eiskïhlung einen allerdings nur geringen Niederschlag, der aber, auf dem Filter gesammelt, die volle Orangefarbe des Arsentrisulfides, mithin eine stattgefundene Reduction von Arsensäure zu arseniger Säure, deutlich darbot.

\section{Versuch.}

Ganz dasselbe Ergebniss hatte ein ebenso angestellter zweiter Versuch, worin die Dialyse fortblieb, und der Schwefelwasserstoff direct in das salzsaure Filtrat eingeleitet wurde.

Diese beiden Versuche sollten Folgendes darthun:

1. Die Reduction geschah auch unter wenig günstigen Umständen, d. h. bei geringer Zerkleinerung' der Leber und bei kurzer Einwirkung. Sie geschah 2. obne dass die Möglichkeit von Fäulniss vorhanden war.

Auf den letzten Punkt hatten wir schon in den vorigen Versuchen geachtet und die Fäulniss abgehalten. In diesen beiden gesehah dies mit besonderer Vorsicht.

Es wurde nun dazu geschritten, den frischen Harn eines Thieres, das Arsen als Trioxyd oder Pentoxyd aufgenommen hatte, auf etwaige Aenderung der Oxyde zu untersuchen. Aaf das Missliche solcher Untersuchung ist schon in der ersten Arbeit iiber unser Thema hingewiesen ${ }^{1}$ ). Die Menge der beiden Arsenverbindungen, die man verfüttern kann, sind wegen ihrer Giftigkeit sehr gering; der Harn scheidet das Arsen nur langsam und spärlich aus; und das böchst complicirte Gemenge, das wir Harn nennen, ist vielleicht für sich selbst im Stande, eine Veränderung, besonders eine Reduction, zu veranlassen.

Am meisten Aussicht, auf diesem Wege eine Ausbeute zu erreichen, gab noch die Arsensäure, weil sie dem Thier in grösserer Gabe beigebracht werden kann als die arsenige Säure. Es wurde deshalb mit ihr begonnen.

\section{Versuch.}

Kaninehen von $1890 \mathrm{~g}$ bekam in 14 Tagen 10 subcutane Einspritzungen von $0,2-0,4$ and schliesslich $0,6 \mathrm{ccm}$ der Natriumarseniatlösung, entspre-

1) Archiv f. exp. Path. u, Pharm. 1879. Bd. XI. S. 212. 
chend den aus der folgenden Uebersicht sich ergebenden Mengen Arsensäure:

$4,6-4,6-9,2-4,6-4,6-4,6-9,2-9,2-9,2-13,8 \mathrm{mg}$ Arsensäure, zusammen $73,6 \mathrm{mg}$.

Nach der 10. Einspritzung verendete das Thier. Die Section ergab nur leichte Anfänge von Gastroenteritis. Es war also eine directe Lähmung der Centren entstanden. Zur Untersuchung gelangten zwei Harnproben, die eine nach der 4. Einspritzung, die andere nach der 10, beide unter dem Siebe, worauf das Thier sass, reinlich aufgesammelt.

Die erste Probe betrug $42,5 \mathrm{ccm}$, war stark alkalisch. Sie wurde unter antiseptischen Maassregeln filtrirt und dialysirt und nach den bekannten Methoden ${ }^{1}$ ) auf arsenige Säure untersucht. Es fand sich gegen $0,1 \mathrm{mg}$ davon. Die Menge der in den Harn übergegangenen Arsensäure war $1,1 \mathrm{mg}$.

Die zweite Probe betrug $52 \mathrm{ccm}$ und reagirte auffallender Weise etwas sauer. Sie wurde ebenfalls unter Zusatz von Chlornatrium und unter Abküblung gegen die doppelte Menge Aussenwasser 27 Stunden lang dialysirt. Das angesäuerte Dialysat gab mit Schwefelwasserstoff in Fiskühlung einen minimalen bräunlichen Niederschlag, der im Marshschen Apparat einen vielleicht $0,1 \mathrm{mg}$ entsprechenden Spiegel lieferte. Aus dem Filtrat schied sich beim Stehen und weiteren Behandeln mit Schwefelwasserstoff in der Wärme ein grauröthlicher Niederschlag aus, der gleich war $0,3 \mathrm{mg}$ Arsensäure. In dieser Harnprobe war also relativ mehr arsenige Säure enthalten als in der ersten.

\section{Versueh.}

Einem Hunde von $9890 \mathrm{~g}$ gaben wir in 19 Tagen mit dem Futter in langsamer Steigerung 145 Tropfen der Natriumarseniatlösung, entsprechend $0,285 \mathrm{~g} \mathrm{As}_{2} \mathrm{O}_{5}$. Das Thier nahm dann kein Futter mehr, wozu die Arsenlösung, selbst in kleinster Menge, zugemischt war. Die nun zur Untersuchung gelangende sauer reagirende Harnprobe maass $75 \mathrm{ccm}$. Sie wurde unter starkem Zusatz von Kochsalz und nnter Abkühlung durch 24 Stunden dialysirt und ergab bei weiterer Behandlung eine ganz geringe Spur arseniger Säure. Das Filtrat gab eine stärkere Spur Arsensäure.

Aus diesem Versuche liess sich demnach nichts folgern; er rechtfertigte nur die Anfangs gegen diesen Gang der Untersuchung geäusserten Bedenken. Das allermeiste der einverleibten Menge von $0,285 \mathrm{~g}$ Arsensäure war entweder noch im Organismus des Hundes aufgespeichert oder war durch den Darm ausgeschieden.

Auch meine andere Vermuthung bestätigte sich, dass nämlich dem Harn allein eine reducirende Kraft gegenüber der Arsensäure zukommen könne. Das ergibt sich aus dem

1) Grabam-Otto-Michaelis, Anorganische Chemie. Bd.II. S. 520. Fresenius, Qualitative Analyse. 1895. S. 453. 


\section{Versuch.}

K a n i n c h e n h a n $70 \mathrm{ccm}$ mit $2,25 \mathrm{ccm}$ Natriumarseniatlösung: $=0,052 \mathrm{As}_{2} \mathrm{O}_{5} 5$ Stunden bei Blutwärme digerirt und gegen $200 \mathrm{ccm}$ Aussenwasser dialysirt. Das mit Salzsäure versetzte Dialysat gab mit Schwefelwasserstoff in der Kälte in 1 Stunde eine allerdings nur minimale unwägbare Abscheidung. Sie war auf dem Filter schmutzig braun, gab aber einen deutlichen Arsenspiegel.

\section{Versuch.}

Kaninchenharn $50 \mathrm{ccm}$, auf Kochsalz aufgefangen, mit $0,25 \mathrm{ccm}$ Natriumarseniatlösung $=0,006 \mathrm{As}_{2} \mathrm{O}_{5}$ ohne vorherige Digestion während 28 Stunden gegen die doppelte Menge Aussenwasser nur dialysirt. Das mit Salzsäure vermischte Dialysat gab mit Schwefelwasserstoff in der Kälte innerhalb 45 Minuten eine deutliche, feinflockige Abscheidung von Arsentrisulfid, die freilich zum Wägen zu gering war.

Besonders der 10. Versuch, worin die quantitativen Bedingungen für das Auffinden des Reductionsproductes sehr günstig waren, zeigt, dass die reducirende Kraft des ungefaulten Kaninchenharnes auf Arsensäure sehr gering ist. Vergleicht man das mit dem Erfolg beim Durchgange der Arsensäure durch den Körper, so erhellt, dass die Reduction hier relativ stärker war.

Die Leber gibt an Wasser Traubenzucker ab, der beim Lieg*en beständig zunimmt. Da nun bei den Versuchen mit der Leber und dem Darm möglicher Weise die Anwesenheit von Zucker die Ursache der starken Reduction sein konnte, so wurde das in Versuch 12 und 13 eigens geprift. Es ergab sich, dass Natriumarseniat durch Traubenzucker unter den hier geltenden Verhältnissen nicht reducirt wird. Ebenso ergab ein Controlversuch, dass Gegenwart von Traubenzucker nicht etwa die Fällung von arseniger Säure durch Schwefelwasserstoff verhindert.

Es schien von Interesse, die etwaige Fähigkeit der Musculatur zum Reduciren der Arsensäure zu untersuchen.

\section{Versuch.}

Der Oberschenkel eines Kaninchens wurde durch einen fest angelegten Gummischlauch und die Hauptgefässe ausserdem noch durch anhaltenden Fingerdruck comprimirt, dann $4,5 \mathrm{ccm}$ Natriumarseniatlösung langsam in die Tiefe der Muskeln des Unterschenkels eingespritzt. Nach 27 Minuten wurde das Thier durch Chloroform getödtet.

Die Muskelpartie war von der eingespritzten Fliissigkeit prall gespannt wie zu Anfang. Das Gewebe wurde mit der Scheere zerschnitten und mit kaltem Wasser ausgezogen. Der Auszug wurde filtrirt, das rothe trübe Filtrat mit Salzsäure versetzt, der dadurch entstandene braune flockige Niederschlag abfiltrirt und das farblose Filtrat unter Eisküh- 
lung mit Schwefelwasserstoff behandelt. Keine Ausfällung von Arsentrisulfid.

Die Muskelsubstanz hatte also unter den angegebenen Verhältnissen keine Arsensäure reducirt.

Dass der Saft des Dünndarmes die Oxydation der arsenigen Säure zu Arsensänre jedesmal prompt vollzieht, habe ich in der vorigen Abhandlung des Näheren beschrieben. Früher schon hatten wir auch die Reduction der Arsensäure beim Aufenthalt im Dünndarm nachgewiesen. Das geschah von Neuem im Folgenden.

\section{Versuch.}

Er wurde an einem kräftigen ätherisirten Kaninchen so ausgefuhrt, wie ich früher mitgetheilt habe; nur wurden, um die Aufsaugung der Lösung im Darm möglichst zu hindern, die Gefässe des Darmstïckes mitabgebunden. Der Darminhalt war vorher durch sanftes Ausstreichen möglichst entfernt. $20 \mathrm{ccm}$ Lösung $=0,460 \mathrm{As}_{2} \mathrm{O}_{5}$ eingespritzt. Das Thier nach 1 Stunde 25 Minuten dureh Chloroform getödtet. Die Darmschlinge war $35 \mathrm{~cm}$ lang, zeigte sich aber, da die Lösung nicht iuberall hin vertheilt war, nur zur Hälfte entzïndet. Der Inhalt wurde sogleich mit Salzsäure ibbersättigt und dann gegen die $4 \frac{1}{1} 2$ fache Menge Aussenwasser 40 Stunden dialysirt. Das mit weiterer Menge Salzsäure vermischte Dialysat gab mit Schwefelwasserstoff in Eiskiuhlung in 1 Stunde eine geringe Abscheidung von schmutzig gefärbtem Arsentrisulfid; das Filtrat davon setzte beim Stehen einen starken Niederschlag von Pentasulfid ab. Quantitativ wurde das Trisulfid bestimmt:

$$
7,3 \mathrm{mg} \quad \mathrm{As}_{2} \mathrm{~S}_{3}=5,9 \mathrm{As}_{2} \mathrm{O}_{3}=6,8 \mathrm{As}_{2} \mathrm{O}_{5} \text {. }
$$

Von der angewandten und dialysirten Arsensäure wurden durch den lebenden Darm in 85 Minuten reducirt 1,5 Proc.

\section{Versuch.}

Starkes Kaninchen. Eingespritzt $10 \mathrm{ccm}$ Natriumarseniatlösung $=$ 0,230 Arsensäure; im Uebrigen alles wie vorher. Das Darmstück war $35 \mathrm{~cm}$ lang und iberall entziindet. Der Inhalt wurde sogleich mit Salzsäure versetzt, filtrirt, das Filtrat diesmal mit Natriumbicarbonat eben übersättigt und nun (statt das vorige Mal sauer, jetzt leicht alkalisch reagirend) gegen die vierfache Menge Aussenwasser 45 Stunden lang dialysirt. Weiter behandelt wie im vorigen Versuche gab es:

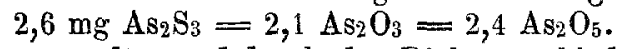

Von der angewandten und durch den Dialysator hindurchgegangenen Arsensäure wurden im lebenden Darm reducirt 1,0 Proc.

Während der Drucklegung warde der unter 12 und 13 erwähnte Versuch auch mit Glykogen angestellt. Gemäss den von ihm bekannten Eigenschaften war von vorneherein nicht wahrscheinlich, dass es die Reduction der Arsensäure zu Stande bringen würde, allein der Sicherheit halber erschien die Prüfung dieser Frage doch erwünscht. Sie wurde genau so angestellt wie mit dem Traubenzucker 
und ergab auch fiur das Glykogen ebenso wenig eine Reduction der Arsensäure wie für jenen.

Als Hauptergebniss der mitgetheilten Versuche gewannen wir somit die Bestätigung, dass frische Theile des Organismus, besonders stark die Leber, ferner der lebende Dünndarm, reducirend auf Arsensäure wirken.

Aufs Neue haben wir gefunden, dass beide Organe in ganz entgegengesetzter Weise reagiren können: auf arsenige Säure oxydirend, auf Arsensäure reducirend.

Mittlerweile hat Professor D. Vitali in Bologna im Anschluss an die von mir und H. Schulz von 1879-1883 in diesem Archive veröffentlichten Versuche die Frage geprüft, ob die arsenige Säure auch beim Durchgehen durch den Organismus oxydirt werde, und hat sie in unserem Sinne beantwortet ${ }^{1}$ ). Er sagt:

„Das Arsenigsäureanhydrid wird im Organismus (des Hundes und des Menschen) zum grössten Theil in Arsensäure umgewandelt, die dann vom Harn als arsensaures Salz ausgeschieden wird."

Die Inangriffnahme desselben Gegenstandes, und zwar sogleich beim Menschen, wird unsere nächste Aufgabe sein.

1) Bollettino chimico-farmaceutico. Mailand 1893. p. 136 und 1896. p. 33. 